\title{
Verapamil in ischaemic heart disease-quantitative assessment by serial multistage treadmill exercise
}

\author{
V. BALASUBRAMANIAN \\ M.D.
}

\author{
G. R. NARAYANAN \\ M.D., D.M.
}

\author{
P. K. KHANNA \\ M.D., D.M. \\ R. S. HooN \\ M.B., F.R.C.P.(Edin)
}

The Unit of Chief Consultant (Medicine), Army Hospital, Delhi Cantt, New Delhi 110010, India

\begin{abstract}
Summary
The efficacy of verapamil in a dose of $120 \mathrm{mg}$ thrice daily was assessed by an objective protocol. Twenty-five patients with ischaemic heart disease were subjected to serial, measured multistage treadmill exercise without any drugs, and after propranolol $\mathbf{4 0} \mathrm{mg}$ t.d.s. for 3 days to assess the stress response to beta-blockade. The tests were repeated after 2,4 and 8 weeks of verapamil. The assessment was based on computerized ST segment changes, the anginal threshold and other objective parameters. Verapamil rendered eight out of thirteen symptomatic patients pain free and significantly increased the anginal threshold in allothers. The ischaemic response as assessed by the ST segment changes on exercise showed a marked improvement in the symptomatic as well as the asymptomatic groups. No major side effects were encountered throughout the study. It was concluded that verapamil in a dose of $360 \mathrm{mg} /$ day is an effective antianginal agent free from significant side effects.
\end{abstract}

\section{Introduction}

The quest for an ideal drug to prevent angina pectoris continues. The long acting nitrates, betablockers and other agents have been tried with conflicting results. Propranolol and its related drugs have become widely accepted despite their limitations.

Verapamil, a papaverine derivative, was introduced in 1962. A number of controlled and uncontrolled clinical trials have shown that the drug ameliorates anginal symptoms in selected groups. Some observers have questioned the validity of the results on the basis of the methods used. Studies by Sandler, Clayton and Thornicroft (1968) and Livesley et al. (1973) have demonstrated its efficacy in a dose of $120 \mathrm{mg}$ thrice daily.

Correspondence: Major V. Balasubramanian, DG's Research Pool Officer. Army Hospital, Delhi Cantt, New Delhi 110010, India.
It is difficult accurately to evaluate the efficacy of an antianginal drug because the most important symptom, pain, is difficult to measure and liable to spontaneous remission. Difficulty in controlling the patient's activity and the element of observer error materially add to these difficulties.

The wide variety of experimental designs such as single-blind, double-blind, double-blind doublecrossover, and double-blind dose-adjusted doublecrossover types have led to confusion, and doubts have been raised concerning the validity of some of the protocols.

Aronow (1973) pointed out that none of the miscellaneous antianginal agents, such as prenylamine, verapamil and perhexilene maleate, is useful even though the original workers have claimed excellent results with these drugs.

In this report the tolerance and electrocardiographic response to exercise in various groups of patients has been assessed. A quantified protocol and an on-line digital computerized ECG monitoring system of known reliability and reproducibility were used (Balasubramanian, Khanna and Hoon, 1975).

\section{Material and methods}

Twenty-five male subjects, aged 34-70 years, attending the Ischaemic Heart Disease Clinic of Army Hospital, Delhi Cantt, were selected for inclusion in this study. They fulfilled the following criteria:

(1) Angina on effort for 6 months or more and developing anginal pain during treadmill exercise (thirteen patients); or history of previous angina and showing classical ischaemic change during exercise (horizontal or down-sloping ST depression of $\geqslant 1.0 \mathrm{~mm}$ ) without developing pain during treadmill exercise (twelve patients);

(2) not receiving any antianginal drugs except glyceryl trinitrite; 
(3) not suffering from any concomitant disease likely to produce false ST changes such as hypertension; (4) free from locomotor disturbances.

\section{Plan of study}

All subjects were evaluated by quantified continuous multistage treadmill exercises according to the following schedule:

(a) Initial evaluation with no drugs; (b) after propranolol $120 \mathrm{mg} /$ day orally for 3 days to assess heart rate and ST segment response to standard dose of a beta-blocking drug; (c) with verapamil $120 \mathrm{mg}$ thrice daily, at 2,4 , and 8 weeks.

Patients were not given any other medication except glyceryl trinitrite sublingually, only if an anginal episode developed.

\section{Quantification of the exercise protocol}

A continuous multistage protocol as shown in Table 1 was used in all subjects (Balasubramanian et al., 1975). The following precautions were observed:

(a) The same set of electrodes with the same electrolyte jelly were used throughout the study with exploring electrode located at V5 position and reference electrode at the manubrium sterni; (b) all studies were conducted in a laboratory (maintained at $28 \pm 2{ }^{\circ} \mathrm{C}$ ) in the post-absorptive state within 2-3 $\mathrm{hr}$ of administration of the last dose in case of verapamil, and 1-2 hr after propranolol; (c) the same timing device accurate to $\pm 5 \mathrm{sec}$ was used during the entire study; (d) the subjects were not allowed to support the weight on the hand rail but could use it for balancing; (e) the speed and gradient of the treadmill were constant; (f) the ECG was fed into an Avionics 2900 stress test monitor with a digital heart rate display, ectopic warning, on-line digital computer module, two-channel oscilloscope and built-in ECG recorder. The heart rate averaged for the last fifteen beats was read from the digital display. The ST segment computer estimated the ST

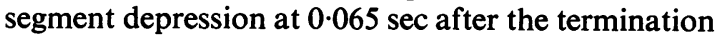
of the $R$ wave in relation to the PR segment in multiples of $0.1 \mathrm{~mm}$.

TABLE 1: Treadmill protocol

\begin{tabular}{lccc}
\hline Gradient & $\begin{array}{c}\text { Speed } \\
(\mathrm{km} / \mathrm{hr})\end{array}$ & $\begin{array}{c}\text { Time } \\
(\mathrm{min})\end{array}$ \\
\hline & $0^{\circ}$ & $2 \cdot 5$ & 2 \\
& $0^{\circ}$ & $5 \cdot 0$ & 1 \\
& $4^{\circ}$ & $5 \cdot 0$ & 3 \\
& $8^{\circ}$ & $5 \cdot 0$ & 3 \\
& $12^{\circ}$ & $5 \cdot 0$ & 3 \\
& $16^{\circ}$ & $5 \cdot 0$ & 3 \\
Extension A & $20^{\circ}$ & $5 \cdot 0$ & 3 \\
Extension B & $20^{\circ}$ & $7 \cdot 0$ & 3 \\
\hline
\end{tabular}

\section{Observations}

The following recordings were made:

(a) Anginal threshold - defined as 'minutes of tread? mill time required to produce definite precordial: pain'; (b) heart rate at rest, at the end of eacto minute during exercise and recovery until it reached control levels; (c) ectopic count per minute; (d£ computer-evaluated ST segment change at sitting and standing postures initially; at the end of eactg minute during exercise; and for $10 \mathrm{~min}$ during recovery. The basal ST levels were deducted from the exercise values to arrive at the net ST change; (eP systolic blood pressure using an aneroid instrumen $\vec{t}$ at the end of each stage of exercise and on terminaes tion.

The exercise was terminated in these patients when. the following were observed:

(a) Anginal pain; (b) $85 \%$ predicted maximal hear rate; (c) extreme fatigue.

The extent of ST segment depression by itself wasnot an indication for stopping the test. No patient had three or more VPB salvos.

\section{Analysis of data}

The following methods of evaluation of the dată were used:

(a) Workload. Calculated cumulatively, eåhe minute's load being worked out as $\mathrm{km} / \mathrm{min}$ walked, multiplied by the gradient of the treadmill in degrees.

(b) ST-segment. The net ST segment displacements per minute is integrated and the total ST change during the entire exercise protocol divided by the corresponding workload.

(c) Anginal threshold. Treadmill time in minutes required to produce anginal pain.

(d) $1 \mathrm{~mm}$ time. Treadmill time in minutes required to produce $1 \mathrm{~mm}$ ST displacement from basal levels.

(e) $2 \mathrm{~mm}$ time. Treadmill time in minutes required to produce $2 \mathrm{~mm}$ ST depression.

(f) Control workload. Identical workload expressed:in minutes of treadmill time as in initial evaluation.

(g) Control heart rate. Heart rates identical with the initial evaluation on exercise.

Standard methods of statistical analysis wereo applied.

\section{Results}

In two of the twenty-five subjects, the propranolol study could not be done owing to certain contra-N indications to beta-blockade. Two-, four- and eight- $\omega$ week studies with verapamil were done in all subjects.

\section{Heart rate response}

The heart rate response is shown in Table 2. The resting heart rate was reduced significantly ${ }^{0}$ $(P=<0.001)$ with propranolol. The reduction $\mathbb{\Phi}$ 
TABLE 2. Mean parameters observed during the trial period

\begin{tabular}{|c|c|c|c|c|c|c|c|c|c|c|c|}
\hline & \multirow[b]{3}{*}{$\begin{array}{c}\text { Resting } \\
\text { heart } \\
\text { rate }\end{array}$} & \multirow[b]{3}{*}{$\begin{array}{c}\text { No developing } \\
\text { angina } \\
(\mathrm{N}=13)\end{array}$} & \multirow{2}{*}{\multicolumn{2}{|c|}{$\begin{array}{l}\text { ST depression } \\
\quad \text { (in } \mathrm{mm})\end{array}$}} & \multirow[b]{3}{*}{$\begin{array}{c}1 \mathrm{~mm} S T \\
\text { time } \\
(\mathrm{min})\end{array}$} & \multirow[b]{3}{*}{$\underset{\text { time }}{2 \mathrm{~mm} \mathrm{ST}}$} & \multirow{2}{*}{\multicolumn{2}{|c|}{ ST index }} & \multirow[b]{3}{*}{ VPB } & \multirow{2}{*}{\multicolumn{2}{|c|}{$\frac{\text { Significance }(P)}{\text { ST index }}$}} \\
\hline & & & & & & & & & & & \\
\hline & & & $\begin{array}{l}\text { Same } \\
\text { work } \\
\text { load }\end{array}$ & $\begin{array}{c}\text { Same } \\
\text { HR }\end{array}$ & & & $\begin{array}{l}\text { Same } \\
\text { work } \\
\text { load }\end{array}$ & Peak & & $\begin{array}{l}\text { Same } \\
\text { work } \\
\text { load }\end{array}$ & Peak \\
\hline Basal & $78 \cdot 6$ & 13 & $2 \cdot 9$ & $2 \cdot 9$ & $4 \cdot 2$ & $6 \cdot 2$ & $11 \cdot 0$ & $11 \cdot 0$ & 120 & - & - \\
\hline $\begin{array}{l}\text { Propranolol } \\
\text { Verapamil }\end{array}$ & $64 \cdot 4$ & - & $1 \cdot 9$ & $2 \cdot 3$ & $7 \cdot 3$ & $11 \cdot 3$ & $3 \cdot 7$ & $6 \cdot 7$ & 70 & $<0.001$ & $<0.001$ \\
\hline 2 weeks & $78 \cdot 0$ & 6 & $1 \cdot 1$ & $1 \cdot 6$ & $12 \cdot 0$ & $18 \cdot 6$ & $1 \cdot 6$ & $2 \cdot 5$ & 18 & $<0.001$ & $<0.001$ \\
\hline 4 weeks & $72 \cdot 2$ & 8 & 0.9 & $1 \cdot 4$ & $12 \cdot 2$ & $18 \cdot 1$ & $1 \cdot 4$ & $1 \cdot 7$ & 22 & $<0.001$ & $<0.001$ \\
\hline 8 weeks & $75 \cdot 6$ & 8 & 0.9 & $1 \cdot 4$ & $13 \cdot 2$ & $18 \cdot 4$ & $1 \cdot 2$ & $1 \cdot 4$ & 20 & $<0.001$ & $<0.001$ \\
\hline
\end{tabular}

TABLE 3. Anginal threshold in minutes in subjects developing pain during initial evaluation

\begin{tabular}{cccccc}
\hline & & & \multicolumn{3}{c}{$\begin{array}{c}\text { Anginal threshold on verapamil } \\
\text { (min) }\end{array}$} \\
\cline { 4 - 6 } Subject & $\begin{array}{c}\text { Age } \\
\text { (years) }\end{array}$ & Basal & 2 weeks & 4 weeks & 8 weeks \\
\hline$(1)$ & 47 & 11 & 15 & No pain & No pain \\
$(2)$ & 42 & 8 & No pain & No pain & No pain \\
$(3)$ & 57 & 9 & No pain & No pain & No pain \\
$(4)$ & 54 & 3 & 11 & 11 & 10 \\
$(5)$ & 52 & 18 & No pain & No pain & No pain \\
$(6)$ & 52 & 10 & No pain & No pain & No pain \\
$(7)$ & 43 & 2 & 5 & 8 & 10 \\
$(8)$ & 34 & 3 & No pain & No pain & No pain \\
$(9)$ & 56 & 17 & No pain & No pain & No pain \\
$(10)$ & 58 & 3 & 10 & 10 & 9 \\
$(11)$ & 60 & 2 & 6 & 10 & 9 \\
$(12)$ & 62 & 2 & 12 & No pain & No pain \\
$(13)$ & 70 & 2 & 7 & 7 & 7 \\
\hline & & & & & \\
\hline
\end{tabular}

with verapamil was less than that produced by propranolol $(P=<0.01)$. Verapamil did not produce a significant reduction compared with propranolol.

\section{Blood pressure}

No changes in supine/standing systolic blood pressure were observed with either verapamil or propranolol. The exercise response was, however, reduced with both the drugs $(P=<0 \cdot 01)$.

\section{Anginal threshold}

The anginal threshold in minutes is given in Table 3. Thirteen patients developed pain during the initial evaluation but eight completed the tests without pain with verapamil therapy. The anginal threshold in the other five subjects was also significantly increased.

\section{$S T$ responses}

The changes in ST segment depression and the ST index and VPB are given in Table 2.

\section{Side effects}

No significant side effects were observed during this trial. Four patients complained of transient constipation. One subject developed urinary retention owing to coexisting prostatic hypertrophy and was relieved by conservative treatment without withdrawal of verapamil. No patient developed AV block, significant hypotension or congestive cardiac failure during the trial.

\section{Discussion}

Hoffman (1964) was the first to report the efficacy of verapamil on the basis of a planned clinical trial. Neumann and Luisada (1966), using a double blind protocol in thirty subjects, concluded that there was a significant decrease in the requirements of nitroglycerine tablets in anginal patients on verapamil. The controversy about the efficacy of the drug began with a review article (Herxheimer, 1967) which stated that 'it has not been established that verapamil is worth considering as an alternative to propranolol; there is some doubt about its efficacy 
and safety'. Hanley and Lebowitz (1967), however, felt that the response to the drug was good to excellent in $70 \%$ of cases and similar reports were published by Misra and Sircar (1967). In another review it was stated that verapamil had been shown in controlled trials to benefit some patients with angina (Today's Drugs, 1968). Subsequently, Phear (1968) condemned the drug on the basis of a doubleblind trial in twenty patients. His views were refuted by Sandler (1968, 1970; Sandler et al., 1968) who published the results of an objective study using 360 $\mathrm{mg}$ of verapamil daily. He found that the effect of the drug was comparable to propranolol in a dose of 300 $\mathrm{mg}$ daily. Hoon (1970) and Banerjee, Mukherjee and Mukherjee (1968) also found the drug to be an effective antianginal agent. In a recent double-blind objective study Livesley et al. (1973) confirmed the earlier views by Sandler (1968). Aronow (1973) focused attention on the limitations of trial protocols and the conflicting reports about verapamil. Krikler (1974) considered that further trials taking into account the weakness in the design of the previous studies were desirable.

Since many workers have demonstrated the efficacy of verapamil as an antianginal agent and the antagonists based their criticism mainly on the trial plans, it was decided to re-evaluate this drug using objective parameters. It had been reported that quantitated treadmill exercises gave reproducible results (Sheffield, 1972; Hartman, Nordstrom and Gobel, 1974). In a preliminary study on anginal patients the authors found that the anginal time, heart rate and ST segment response could accurately be reproduced. The evaluation of pain and the need for nitroglycerine tablets have been reported to yield unreliable results owing to the fluctuation in the patient's activity, environment and metabolic needs (Gettes, 1974). It has been reported that the majority of patients with angina pectoris and positive stress tests have episodes of 'painless' ST depression (Wolf, Tzivoni and Stern, 1974). The pain threshold and the influence of physical and psychic factors are individual characteristics and may affect chest pain. Gettes (1974) suggested the use of parameters of myocardial ischaemia which are more objective than chest pain. He thought that exercise stress testing is the simplest method available to evaluate myocardial ischaemia. These considerations lent further support to the trial plan used.

In this study a placebo protocol was not used. The subject acted as his own control and observer-bias was minimized by comparable tests (Hartman, Nordstrom and Gobel, 1974). Objective data for a variable protocol $(1 \mathrm{~mm}, 2 \mathrm{~mm}$ time, $S T$ index, and anginal threshold) were used to correlate exercise with the ischaemic response. The post-exercise electrocardiograms, although recorded, were not included, as a reliable method of correlating these changes with variable exercise has not yet been evolved.

Propranolol was used in a relatively small dose of $\stackrel{c}{.}$ $120 \mathrm{mg}$ daily even though workers from western $\overrightarrow{\overrightarrow{\vec{S}}}$ countries have used greater doses. The intention was $\overrightarrow{0}$ to study the physiological response of the patients to $\frac{C}{0}$ stress under beta-blockade and not to compare the $\frac{\bar{\omega}}{\bar{s}}$ efficacy of propranolol to verapamil as an anti- $\widetilde{\nabla}$ anginal agent. The efficacy of propranolol in a greater dose and its comparison with verapamil has already is been extensively reported (Sandler, 1968; Livesley $\vec{\circ}$ et al., 1973).

Verapamil did not affect the resting heart rate, $\vec{\omega}$ but significantly decreased the exercise heart rate. In $\overline{0}$ most of the subjects the ST response markedly 3 improved in spite of the exercise load being increased. iv The subjects invariably showed an increase in anginal $N$ threshold, the peak effect being observed at 4 weeks. The exercise ventricular ectopic count was also significantly reduced. The incidence of complications $\vec{\omega}$ was minimal and did not necessitate withdrawal of the drug or reduction in its dosage. In two cases who could not be given propranolol owing to coexistent $\bar{z}$ bronchospasm, verapamil did not aggravate the $\frac{\stackrel{0}{O}}{3}$ symptoms. No instance of congestive cardiac failure $\mathcal{S}$ was encountered in this group.

\section{Conclusions}

Verapamil in a dose of $120 \mathrm{mg}$ t.d.s. is an effective antianginal agent as demonstrated objectively in subjects with ischaemic heart disease. It increases anginal threshold in the severe cases, eliminates pain $\stackrel{\varnothing}{\varnothing}$ in the moderate cases and improves the ischaemic $\overrightarrow{\vec{F}}$ response in all cases, and causes no significant side effects.

\section{References}

Aronow, W.S. (1973) Medical treatment of angina pectoris, VIII. Miscellaneous antianginal drugs. American $\bar{\sigma}$ Heart Journal, 85, 132.

Balasubramanian, V., Khanna, P.K. \& Hoon, R.S. (1975) On-line digital computer quantitated ST segment response to sub-maximal treadmill exercise. Journal of the Association of Physicians of India, 23, 1.

BANERJEe, J.C., MukHERJEe, T.P. \& MukHERJEe, S.K. (1968) Verapamil hydrochloride in angina pectoris. Journal of Indian Medical Association, 51, 281.

Getres, S.L. (1974) Painless myocardial ischaemia. Chest, 66, 612.

HaNLey C. \& Lebowitz W.B. (1967) Iproveratril in angina $N$ pectoris. St Vincent's Hospital Medical Bulletin, $2,1$.

Hartman, K.E., Nordstrom, L.A. \& Gobel, F.L. (1974) The effect of placebo therapy on exercise response and $\sigma$ nitroglycerine consumption in patients with angina pectoris. Circulation (Abst.), (453) 50, 115.

HeRXHEIMER, A. (1967) Claims for cordilox. Drug and $\mathbb{D}$ Therapeutic Bulletin, 5, 85.

HoFfmaN, P. (1964) Treatment of coronary circulatory drugs with Isoptin in general practice. Medizinische Klinik, 59, 1387. 
Hoon, R.S. (1970) Coronary vasodilators-Iproveratril. A clinical trial. Indian Journal of Medical Sciences, 24, 119. KRIKLER, D. (1974) Verapamil in cardiology. European Journal of Cardiology, 2/1, 3.

Livesley, B., Catley, P.F., Campbell, R.C. \& Oram, S. (1973) Double blind evaluation of verapamil, propranolo and isosorbide dinitrate against a placebo in the treatment of angina pectoris. British Medical Journal, 1, 375.

MisRA, S.S. \& SiRCAR, A.R. (1967) Iproveratril-A new coronary dilator. Journal of Indian Medical Association, 48, 163.

Neumann, M. \& Luisada, A.A. (1966) Double-blind evaluation of orally administered iproveratril in patients with angina pectoris. American Journal of Medical Sciences, 251, 55.

Phear, D.N. (1968) Verapamil in angina. British Medical Journal, 2, 740.
SANDler, G. (1968) Verapamil in angina. British Medical Journal, 3. 56.

SANDLER, G. (1970) Investigation of verapamil in the treatment of angina pectoris. Bruxelles médical, 50, 669.

Sandler, G., Clayton, G.A. \& Thornicroft, S.G. (1968) Clinical evaluation of verapamil in angina pectoris. British Medical Journal, 3, 224.

SHEFFIELD, L.T. (1972) Quantitative approach to exercise testing for ischaemic heart disease. In: Quantitation in Cardiology, 1st Edn. Leiden University Press: The Netherlands.

ToDAY's Drugs (1968) Verapamil. British Medical Journal, 1, 230.

Wolf, E., Tzivoni, D. \& Stern, S. (1974) Comparison of exercise test and 24-hour ambulatory electrocardiographic monitoring in detection of ST-T changes. British Heart Journal, 36, 90 . 